\title{
Derivation of orbital parameters of very low mass companions in double stars from radial velocities and observations of space astrometry missions like HIPPARCOS, DIVA and GAIA
}

\author{
H.-H. Bernstein \\ Astronomisches Rechen-Institut, Mönchhofstraße 12 -14, D-69120 \\ Heidelberg, Germany
}

\begin{abstract}
Radial velocity measurements are a well known high-precision method to obtain the orbits of extrasolar planets or brown dwarfs. However, this method is not able to determine the inclination which could be derived from astrometry. The astrometric effects of those objects are very minute, wherefore the interest of astronomers in astrometric techniques was very poor. This situation changes fundamentally since space astrometry observations are available. HIPPARCOS demonstrated the power of space astrometry and the extremely high accuracy of the DIVA, and especially the GAIA observations allows one to detect Jupiter- and Earth- like objects. The optimal estimation of the parameters of the orbit of extrasolar planets or brown dwarfs is a combination of radial velocity measurements and space astrometry observations. Here it is possible to overcome problems which are inherent in both observation methods, so space astrometry complements radial velocity observations and vice versa. This paper gives a method for the parameter estimation using both types of measurements.
\end{abstract}

\section{Introduction}

Up to now, astrometry was not able to give any considerable contribution to the detection of (very) low mass companions in double star systems. The accuracy of classical astrometric techniques does not allow to catch the astrometric effect of such a double star system. Here, the typical size of the wobble of the photocenter is of the order of a few milliarcseconds and in the case of extrasolar planets of about some microarcseconds (Gliese 1982). This situation has been changed now fundamentally! Apart of ground based interferometer observations, the astrometric satellite projects of the European (\& German) space agency will be able to overcome this accuracy defile.

\section{The capability of space astrometry}

Since one year ago, the HIPPARCOS project is terminated (ESA 1997) and the relevant results are condensed in Table 1 , together with the goals of the next astrometric milestone, GAIA. It's planned to combine the astrometric observ- 

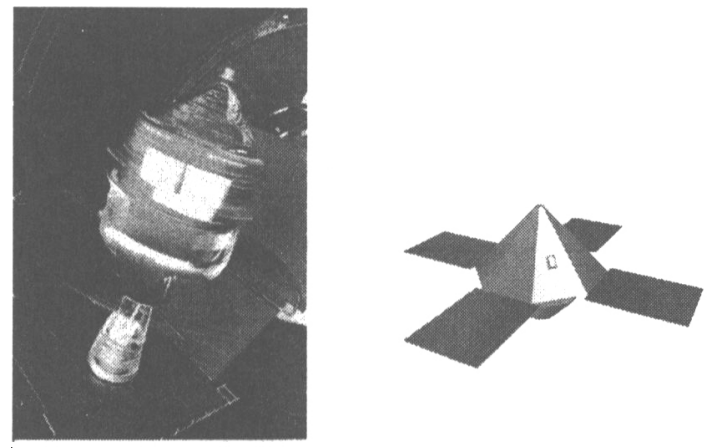

DIVA is a German precursor mission to GAIA with the same measurement techniques: a Fizeau interferometer on a small satellite to perform astrometric and photometric measurements. Here, the achievable accuracy will be a factor of three better than HIPPARCOS.

Figure 1. HIPPARCOS photo and DIVA model

ing facility with radial velocity measurement equipment. Due to the observing techniques, especially the scanning mode of such an astrometric satellite, those measurements have an accuracy of about $1-10 \mathrm{~km} / \mathrm{s}$. This is no appropriate tool for searching of low mass companions. One gets additional information for stellar dynamics only.

Table 1. Measurement Goals

\begin{tabular}{crrr} 
Parameter & HIPPARCOS & DIVA & GAIA \\
\hline No. of Objects & 120000 & 1000000 & 50000000 \\
No. of Objects & $1000000^{*}$ & - & - \\
Lim. Mag. & $\mathrm{V}=12 \mathrm{mag}$ & $\mathrm{V} \geq 12 \mathrm{mag}$ & $\mathrm{V} \geq 15.5 \mathrm{mag}$ \\
Poss. Acc. & $1-2 \mathrm{mas}$ at $\mathrm{V}=10$ & $0.8 \mathrm{mas}$ at $\mathrm{V}=12$ & $10 \mu \mathrm{as}$ at $\mathrm{V}=15$ \\
Pr. Mo. Acc. & $1-2 \mathrm{mas} / \mathrm{y}$ at $\mathrm{V}=10$ & $1.0 \mathrm{mas} / \mathrm{y}$ at $\mathrm{V}=12$ & $10 \mu \mathrm{as} / \mathrm{y}$ at $\mathrm{V}=15$ \\
Duration & 3.1 years & 2 years & 5 years \\
\hline
\end{tabular}

*Tycho catalogue 1

The mission lifetime limits the detectability of low mass companions in general, but a combination of two or more astrometric missions improves this situation fundamentally. Depending on the scanning law, the shortest period is about 30 days for all three satellite projects and the longest period is about 1.5 times the observing time span. In general, the observed periods are longer in astrometry than usually detected with radial velocity measurements.

On the basis of Kepler's law Figs. 2, refdivlim and 4 show the capability of these satellite projects concerning the detection of low-mass companions plotted versus a reasonable range of period in a dynamical mass determination. For HIPPARCOS, DIVA \& GAIA we have a $3 \sigma$ limit for the semi-major axis of 6 mas, 2.4 mas \& $30 \mu a s$. Within these figures one has two curves, the upper one is calculated for a G2 star at a distance of 40pc (GAIA: 200pc) as primary component the lower for a M0 star at a distance of $10 \mathrm{pc}$. In the case cof HIPPARCOS one can agree that the detection of very low mass companions

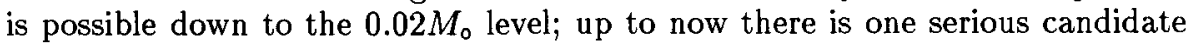
(Bernstein 1997). In the case of GAIA we expect 10000000 candidate stars 


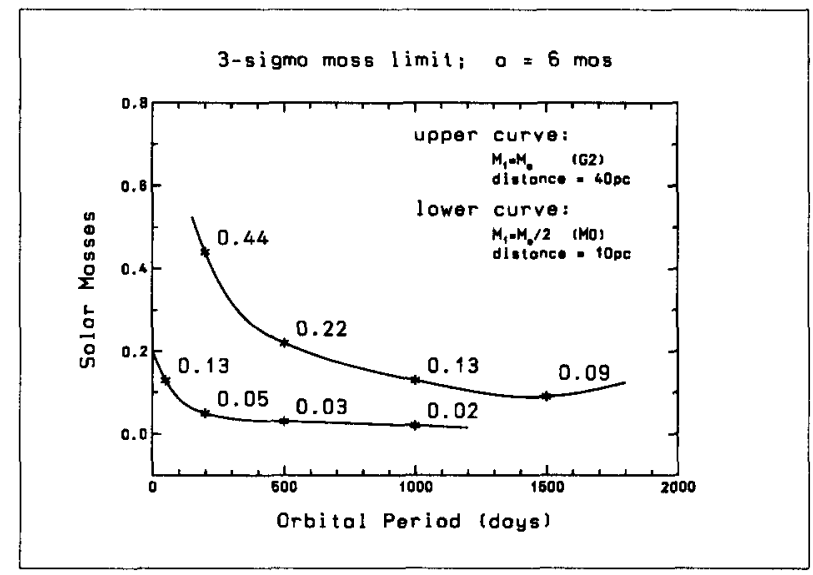

Figure 2. HIPPARCOS low-mass detection capability

with a brown dwarf as companion. Furthermore, we expect to investigate about 1000000 stars probably orbited by a Jupiter like planet and 150 stars orbited by an Earth like planet (Casertano, Lattanzi, \& Perryman 1995).

\section{The astrometric detection procedure}

The detection procedure starts with the augmentation of the well known classical observational equations (Bernstein 1994) by terms of the orbital motion. For the description of the orbital motion, one should use the Thiele-Innes constants for the location of the orbit in space and the Fourier series of the elliptical coordinates in the orbital plane (Bernstein \& Bastian 1995). So one has a linear observational equation of the observed quantity, the abscissae $\psi$, and orbital parameters together with the classical astrometric parameters $(\alpha, \delta, \mu \alpha, \mu \delta$ $\& \pi^{*}$ ), where the period of the double star system is a variable parameter. Accomplishing the parameter estimation by variing the period in reasonable limits, one derives the Fig. 5, where the minimum of the rms of unit weight or the maximum of the signal to noise ratio of the semi-major axis of the derived solution indicates the best result of this parameter estimation.

The results have to satisfy some plausibility constraints like first harmonic larger than the second one and so on. At least we use these results as starting values for an iterative least squares adjustment like the Levenberg Marquardt algorithm for the combined parameter estimation with the classical Kepler elements as the orbital parameters.

On the microarcsecond level, especially for observing periods larger than 4 years, we have to introduce second order effects like the foreshortening of the orbital parameters. It's a well known effect with well known formulas and simple to use during the classical double star data reduction. A problem arises when we consider the detection procedure - this effect is no more negligible. In the future the Fourier coefficients in the equations of orbital motion are no longer constant but varying with time. I think one can tackle this problem by the utilisation 


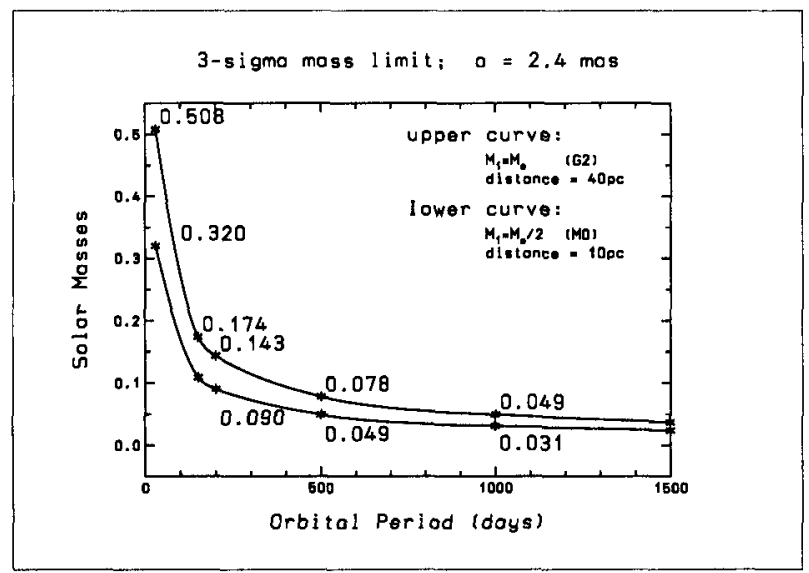

Figure 3. DIVA low-mass detection capability

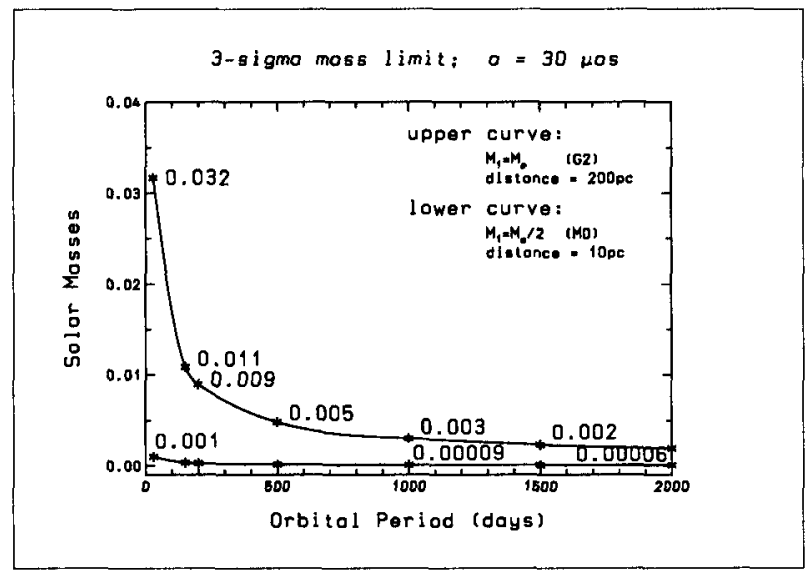

Figure 4. GAIA low-mass detection capability
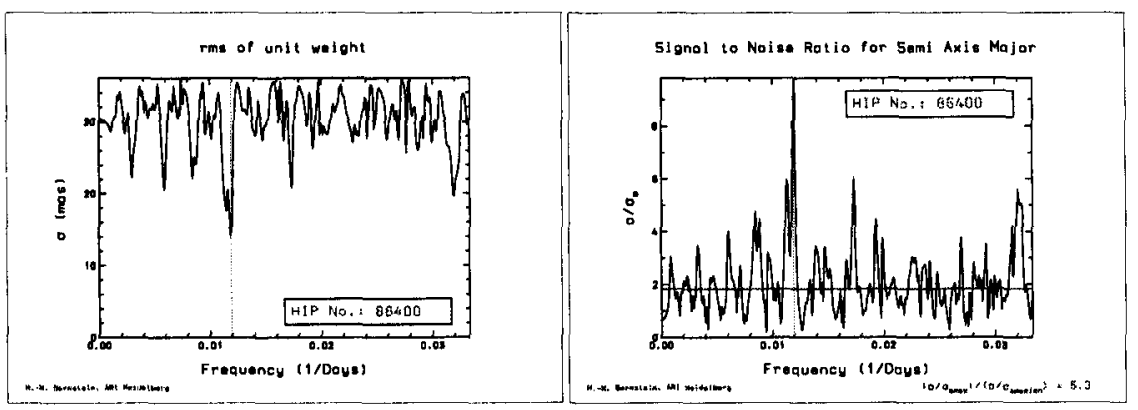

Figure 5. Left: rms of unit weight, and right: signal-to-noise ratio, of the semi-major axis for Gliese 688 
of an evolutionary spectra, or more sophisticated by a wavelet transformation, here the zoom effect is important. The Morlet wavelet seems to be a good mathematical basis of this work.

The problems of space astrometry in this case are concerned with well known problems of one dimensional, unevenly spaced data, which are delivered by the scanning law of the satellite. From time series analysis we know the aliasing effect or how to catch the correct order of harmonic. Due to a limited number of observations one is not able to use the full elliptical parametrization and the fit of a circle in space must be contended. In general, the law of areas seperates the inclination effect from the effect of ellipticity. Sometimes the time distribution of such a scanning satellite does not allow one to benefit from this basic fact. Depending on the quality of the starting values, one could get problems of the convergence of the Levenberg Marquardt adjustment algorithm.

\section{The combined parameter estimation}

In principle, astrometry observes the motion of the photocentre of a double star system in question. The observational equation concerning the measured abscissae $\psi$ embraces all parameters of the orbit:

$$
\psi=\psi[\underbrace{\alpha, \beta, \mu \alpha, \mu \beta, \pi^{*} ; a, \Omega, i, \omega, \boldsymbol{e}, T, P}_{: \mathbf{x}} ;(\Delta m) ; \mathbf{T}]
$$

Usually radial velocity measurements gives the parameters which are condensed here in a vector $y$ whereas one calculates the corresponding covariance matrix $\mathbf{Q}_{\mathbf{y}}$ which has a simple diagonal structure, normally:

$$
V=V[\gamma ; \underbrace{a_{1,2} \cdot \sin i, \omega, e, T, P}_{\hookrightarrow \hat{\mathbf{y}} \& \mathbf{Q}_{\mathbf{y}}}]
$$

For a combined least squares parameter estimation we have two types of observational equations:

$$
\begin{array}{ccc}
\mathbf{l}_{\psi}+\mathbf{v}_{\psi}=\mathbf{A} \cdot \mathbf{x} & ; & \mathbf{l}_{y}+\mathbf{v}_{y}=\mathbf{B} \cdot \mathbf{x} \\
\mathbf{A}=\frac{\partial \psi}{\partial x} \quad ; \quad \mathbf{C}_{\psi}=\left[\frac{\sigma_{\psi}}{\sigma_{\diamond_{\psi}}}\right]^{2} & ; \quad \mathbf{B}=\frac{\partial y}{\partial x} \quad ; \quad \mathbf{C}_{y}=f \cdot \mathbf{Q}_{y}
\end{array}
$$

$\mathbf{l}_{\psi}$ and $\mathbf{l}_{y}$ are the vectors of the observed minus computed quantity, $\mathbf{v}_{\psi}$ and $\mathbf{v}_{y}$ are the vectors of the corresponding residuals furthermore $\mathbf{A}$ and $\mathbf{B}$ are the coefficient matrices containing the needed partial derivatives. Finally, $\mathbf{C}_{\psi}$ and $\mathbf{C}_{y}$ are the used covariance matrices with $f=1$ normally. In this context it is clear that $\mathbf{B}$ is a sparse matrix with the consequence, that the corresponding part of the normal equation matrix $\mathbf{B}^{T} \mathbf{C}_{y}^{-1} \mathbf{B}$ cannot be positive definite.

Using the norm (5) one gets with (3) and (4) the parameter estimation (6), embracing all the parameters of the double star system described in (1) and (2):

$$
\begin{gathered}
\mathbf{v}_{\psi}^{T} \mathbf{C}_{\psi}^{-1} \mathbf{v}_{\psi}+\mathbf{v}_{y}^{T} \mathbf{C}_{y}^{-1} \mathbf{v}_{y}=\min \\
\widehat{\mathbf{x}}=\left(\mathbf{A}^{T} \mathbf{C}_{\psi}^{-1} \mathbf{A}+\mathbf{B}^{T} \mathbf{C}_{y}^{-1} \mathbf{B}\right)^{-1} \cdot\left(\mathbf{A}^{T} \mathbf{C}_{\psi}^{-1} \mathbf{l}_{\psi}+\mathbf{B}^{T} \mathbf{C}_{y}^{-1} \mathbf{l}_{y}\right)
\end{gathered}
$$




\subsection{The minimum demand $f$ on radial velocity observations}

If one uses a scanning satellite like HIPPARCOS or GAIA for astrometric measurements, one gets a fixed frame of observations for all stars. One gets a certain number of measurements with a fixed time distribution of observational epochs, together with a fixed rms of unit weight which depends on the capability of the instrument. And one has a fixed covariance matrix of the observations and a fixed coefficient matrix A. For radial velocity measurements, this situation is totally different. If there is any kind of ambiguity, or any insufficient measurement accuracy, we are able to record one or more spectra of an object in question. If we plan to combine space astrometry with radial velocity observations by a new series of measurements, one should ask for an optimal time distribution and a minimum number of spectra to insure an adaquate accuracy balance. For this reason I introduced the parameter $f$ in (4). In order to estimate an optimal value of $f$, one should minimise the trace or the determinant of the covariance matrix of the unknowns:

$$
\begin{aligned}
& \text { trace }\left|\left(\mathbf{A}^{T} \mathbf{C}_{\psi}^{-1} \mathbf{A}+\mathbf{B}^{T} \mathbf{C}_{y}^{-1}(f) \mathbf{B}\right)^{-1}\right|=\min \\
& \operatorname{det}\left|\left(\mathbf{A}^{T} \mathbf{C}_{\psi}^{-1} \mathbf{A}+\mathbf{B}^{T} \mathbf{C}_{y}^{-1}(f) \mathbf{B}\right)^{-1}\right|=\min
\end{aligned}
$$

Using $f$, it is easy to derive an appropriate time distribution of $V$ or the minimum number of spectra.

Acknowledgments. The author is indebted to thank Dr. U. Bastian for fruitful discussions about astrometry and radial velocity measurements.

\section{References}

Bernstein, H.-H. 1994, A\&A, 283, 293

Bernstein, H.-H. 1997, in Hipparcos Venice '97 (ESA SP-402), B. Battrick, M.A.C. Perryman, \& P.L. Bernacca, Noordwijk: European Space Agency, 705

Bernstein, H.-H., \& U. Bastian 1995, in Future Possibilities for Astrometry in Space (ESA SP-379), M.A.C. Perryman \& F. van Leeuwen, Noordwijk: European Space Agency, 55-59

Casertano, S., Lattanzi, M.G. \& Perryman, M.A.C. 1995, in Future Possibilities for Astrometry in Space (ESA SP-379), M.A.C. Perryman \& F. van Leeuwen, Noordwijk: European Space Agency, 47-54

Gliese, W. 1982, in Scientific Aspects of the Hipparcos Space Astrometry Mission (ESA SP-177), M.A..C. Perryman \& T.D. Guyenne, Noordwijk: European Space Agency, 193

ESA 1997, The Hipparcos and Tycho Catalogues, ESA SP-1200 